\title{
openheart Ferumoxytol-enhanced MRI in patients with prior cardiac transplantation
}

Colin G Stirrat $(1),{ }^{1}$ Shirjel Alam, ${ }^{2}$ Thomas J MacGillivray, ${ }^{3}$ Calum Gray, ${ }^{3}$ Marc Richard Dweck, ${ }^{1}$ Victor Jones, ${ }^{1}$ William Wallace, ${ }^{4}$ John R Payne, ${ }^{5}$ Sanjay K Prasad, ${ }^{6}$ Roy S Gardner, ${ }^{7}$ Mark C Petrie, ${ }^{8,9}$ Saeed Mirsadraee, ${ }^{3}$ Peter Henriksen, ${ }^{10}$ David E Newby, ${ }^{1}$ Scott Semple ${ }^{3}$

To cite: Stirrat CG, Alam S, MacGillivray TJ, et al. Ferumoxytol-enhanced MRI in patients with prior cardiac transplantation. Open Heart 2019;6:e001115. doi:10.1136/ openhrt-2019-001115

Received 13 June 2019 Revised 10 July 2019 Accepted 11 September 2019

\section{Check for updates}

(c) Author(s) (or their employer(s)) 2019. Re-use permitted under CC BY. Published by BMJ.

${ }^{1}$ Centre for Cardiovascular Science, University of Edinburgh, Edinburgh, UK

${ }^{2}$ Department of Cardiovascular Sciences, University of Edinburgh, Edinburgh, UK ${ }^{3}$ Clinical Research Imaging Centre, University of Edinburgh, Edinburgh, UK

${ }^{4}$ Department of Pathology, University of Edinburgh,

Edinburgh, UK

${ }^{5}$ Scottish National Advanced Heart Failure Service (SNAHFS), Golden Jubilee National Hospital, Clydebank, UK

${ }^{6}$ Department of Cardiology, Royal Brompton Hospital, London, UK

${ }^{7}$ Scottish Advanced Heart Failure Unit, Golden Jubilee National Hospital, Clydebank, UK

${ }^{8}$ Institute of Cardiovascular and Medical Sciences, University of Glasgow, Glasgow, UK

${ }^{9}$ Golden Jubilee National Hospital, Clydebank, UK

${ }^{10}$ Edinburgh Heart Centre, Royal Infirmary of Edinburgh, Edinburgh, UK

Correspondence to Dr Colin G Stirrat; colin.stirrat@ ed.ac.uk

\section{ABSTRACT}

Objectives Ultra-small superparamagnetic particles of iron oxide (USPIO)-enhanced MRI can detect cellular inflammation within tissues and may help non-invasively identify cardiac transplant rejection. Here, we aimed to determine the normal reference values for USPIOenhanced MRI in patients with a prior cardiac transplant and examine whether USPIO-enhanced MRI could detect myocardial inflammation in patients with transplant rejection.

Methods Ten volunteers and 11 patients with cardiac transplant underwent $\mathrm{T} 2, \mathrm{~T}^{*}$ and late gadolinium enhancement 1.5T MRI, with further $\mathrm{T}^{*}$ imaging at 24 hours after USPIO (ferumoxytol, $4 \mathrm{mg} / \mathrm{kg}$ ) infusion, at baseline and 3 months.

Results Ten patients with clinically stable cardiac transplantation were retained for analysis. Myocardial T2 values were higher in patients with cardiac transplant versus healthy volunteers $(53.8 \pm 5.2$ vs $48.6 \pm 1.9 \mathrm{~ms}$, respectively; $p=0.003$ ). There were no differences in the magnitude of USPIO-induced change in $\mathrm{R}^{*}$ in patients with transplantation (change in $\mathrm{R} 2{ }^{*}, 26.6 \pm 7.3$ vs $22.0 \pm 10.4 \mathrm{~s}^{-1}$ in healthy volunteers; $p=0.28$ ). After 3 months, patients with transplantation $(n=5)$ had unaltered T2 values $(52.7 \pm 2.8$ vs $52.12 \pm 3.4 \mathrm{~ms} ; \mathrm{p}=0.80$ ) and changes in R2* following USPIO (29.42 \pm 8.14 vs $25.8 \pm 7.8 \mathrm{~s}^{-1} ; \mathrm{p}=0.43$ ).

Conclusion Stable patients with cardiac transplantation have increased myocardial T2 values, consistent with resting myocardial oedema or fibrosis. In contrast, USPIO-enhanced MRI is normal and stable over time suggesting the absence of chronic macrophage-driven cellular inflammation. It remains to be determined whether USPI0-enhanced MRI may be able to identify acute cardiac transplant rejection.

Trial registration number NCT02319278349 (https:// clinicaltrials.gov/ct2/show/NCT02319278) Registered 03.12.2014 EUDraCT 2013-002336-24.

\section{INTRODUCTION}

Cardiac transplantation is a life-prolonging treatment for end-stage cardiac disease. Transplant rejection is a major threat to the allograft, requiring treatment in around one in eight transplant recipients in the first year ${ }^{1}$ but can occur at any stage after

\section{Key questions}

What is already known about this subject?

- Ultra-small superparamagnetic particles of iron oxide (USPIO) are ingested by tissue macrophages that can be visualised using MRI to highlight areas of macrophage inflammation within the heart.

What does this study add?

- Stable patients with cardiac transplantation have increased myocardial T2 values, consistent with resting myocardial oedema or fibrosis. Despite this, USPIO-enhanced MRI is normal and stable over time suggesting the absence of chronic macrophage-driven cellular inflammation.

How might this impact on clinical practice?

- USPIO-enhanced T2* MRI may still prove to be of value in diagnosing and monitoring conditions with macrophage-driven myocardial inflammation, including acute transplant rejection with associated macrophage infiltration.

transplantation and causes significant morbidity and mortality. Rejection is notoriously difficult to diagnose using existing non-invasive imaging methods with repeated surveillance myocardial biopsies often undertaken.

Most cases of acute rejection are due to cellular rejection with antibody-mediated rejection less prevalent. Rejection severity is classified according to histological findings, and although the cellular infiltrate in acute cellular rejection is predominantly lymphocytic, macrophage infiltration has a key role. ${ }^{23}$ The importance of macrophages in acute cardiac allograft rejection was recently emphasised in a rodent study that showed depletion of circulating macrophages protected the allograft against rejection, raising the possibility of therapeutic targeting of macrophages as a novel treatment strategy.

Iron oxide nanoparticles are generating interest as a MRI contrast medium that is able to detect macrophages, and clinical 
applications, such as myocardial infarction, are now emerging. ${ }^{5-11}$ Ultra-small superparamagnetic particles of iron oxide (USPIO) consist of an iron oxide core surrounded by a carbohydrate or polymer coating. They are small enough to extravasate passively through capillaries, where they are engulfed by tissue-resident macrophages ${ }^{12}$ and are detectable by T2*-weighted MRI. Thus, USPIO-enhanced MRI can identify tissue-resident macrophage activity and help to identify cellular inflammation within tissues.

Promising preclinical studies have shown USPIO-enhanced MRI is able to detect acute cardiac and renal allograft rejection with USPIO signal correlating with macrophage distribution, rejection severity on histology and impaired cardiac function. Moreover, this approach can also be used to assess treatment response with rodent models demonstrating less USPIO enhancement following initiation of immunosuppression. ${ }^{413-17}$ A future role of USPIOs includes a 'theranostic' strategy whereby imaging is combined with therapy; Guo et al ${ }^{18}$ recently conjugated an iron nanoparticle to a CD-3 antibody and a therapy gene, allowing imaging and targeting of $\mathrm{T}$ cells that play a central role in acute cardiac allograft rejection.

In this study, we aimed to assess and quantify myocardial USPIO enhancement in stable patients with cardiac transplantation and patients with cardiac transplant rejection, correlating enhancement with clinical measures of inflammation and oedema including T2 mapping MRI,a quantitative imaging method assessing myocardial oedema in transplant rejection. ${ }^{19}{ }^{20}$ We hypothesised that USPIO-enhanced MRI would detect myocardial macrophage activity in the inflamed myocardium of rejecting transplanted hearts, but not in stable healthy cardiac allografts, and provide a cellular-specific non-invasive imaging technique that may aid and improve patient diagnosis and management.

\section{METHODS}

This was an open-label observational multicentre cohort study (NCT02319278) that recruited patients between January 2015 and May 2016. The study was performed in accordance with the Declaration of Helsinki, the approval of the Scotland A Research Ethics Committee (13-SS0111), and the written informed consent of all participants. The Medicines and Healthcare products Regulatory Authority of the United Kingdom gave Clinical Trial Authorisation for the study (EUDraCT 2013-002336-24).

\section{Study populations}

Adult ( $>18$ years of age) patients with a history of cardiac transplantation (including suspected allograft rejection) were recruited into the study. Healthy volunteers had no clinically significant medical history. Exclusion criteria were contraindication to MRI or ferumoxytol infusion, any other inflammatory comorbidity, renal failure (estimated glomerular filtration rate $<30 \mathrm{~mL} / \mathrm{min} / 1.73 \mathrm{~m}^{2}$ ), pregnancy, breastfeeding and women of childbearing potential without reliable contraception.

\section{Study protocol}

Patients with cardiac transplantation and healthy volunteers underwent paired MRI scans at baseline, and patients were invited to return for repeat imaging after 3 months. At the time of scanning, blood samples were collected for clinical haematology and biochemistry measurements.

\section{Magnetic resonance imaging}

MRI was performed using a MAGNETOM Avanto 1.5T MRI (Siemens Healthcare, Erlangen, Germany), with a dedicated cardiac array coil. All images were acquired with ECG gating using expiration breath-holds. Routine steady state free precession (TrueFISP) sequences were used to acquire long-axis and short-axis cine images of the heart (repetition time (TR) $85.8 \mathrm{~ms}$, echo time (TE) $1.45 \mathrm{~ms}$, flip angle $50^{\circ}$, matrix $173 \times 256$, field of view (FoV) $400 \mathrm{~mm}$, slice thickness $8 \mathrm{~mm}, 2 \mathrm{~mm}$ gap). Quantitative USPIO imaging was performed in similar slice positions using a prototype T2*-weighted multigradient-echo acquisition with a volumetric shim applied over the entire heart volume (TR $996 \mathrm{~ms}$, TE 2.13, 4.3, 6.4, 8.6, 10.7, 12.8, 15.0, $17.1 \mathrm{~ms}$, flip angle $18^{\circ}$, matrix $130 \times 256$, FoV $400 \mathrm{~mm}$, slice thickness $6 \mathrm{~mm}$, gap $4 \mathrm{~mm}$ ). The T2*-weighted acquisitions included views through the liver, spleen and spine to allow quantification of USPIO accumulation within organs of the reticuloendothelial system. The same T2* protocol was used to quantify USPIO accumulation 24 hours after infusion allowing calculation of T2* relaxation rates before and after administration of USPIO. T2 mapping was conducted using a Siemens prototype T2-prepared TrueFISP acquisition acquiring identical long-axis and short-axis slice positions (TR $219.3 \mathrm{~ms}$, TE $1.07 \mathrm{~ms}$, T2 prep durations $0,25,50 \mathrm{~ms}$, flip angle $70^{\circ}$, matrix 130×192, FoV $400 \mathrm{~mm}$, slice thickness $6 \mathrm{~mm}, 4 \mathrm{~mm}$ gap). T2P-TrueFisp images are acquired at intervals of at least three RR intervals to allow for sufficient magnetisation recovery in between acquisitions.

Immediately after the baseline $\mathrm{T} 2$ and $\mathrm{T} 2{ }^{*}$-weighted scan, participants received an intravenous administration of gadolinium contrast medium $(0.15 \mathrm{mmol} / \mathrm{kg}$; Gadovist, Bayer Plc, Germany) followed by breath-held inversion recovery sequences in long-axis and short-axis planes to acquire late-enhancement images. Optimal inversion time (TI) was determined on a slice-by-slice basis using standard late-enhancement TI-scout protocols (TR $750 \mathrm{~ms}$, TE $2.61 \mathrm{~ms}$, flip angle $20^{\circ}$, matrix $173 \times 256$, FoV $400 \mathrm{~mm}$, slice thickness $9 \mathrm{~mm}$, gap $1 \mathrm{~mm}$ ). The inversion-recovery late-enhancement short-axis slices were acquired using similar slice positions as the T2-oedema and $\mathrm{T} 2 *$-weighted imaging.

\section{Ultra-small superparamagnetic particles of iron oxide}

Intravenous infusion of USPIO (ferumoxytol, $4 \mathrm{mg} / \mathrm{kg}$; Rienso, Takeda Italia, Italy) was performed immediately 
$\mathrm{R} 2 *$ map
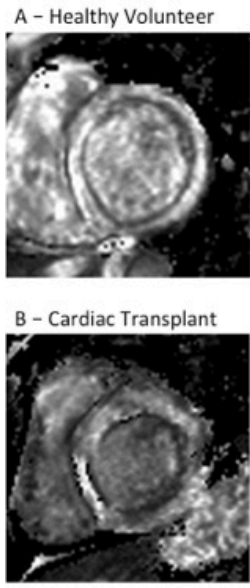

C - Myocardial Infarction

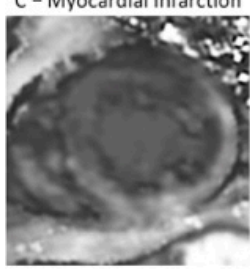

T2 Map
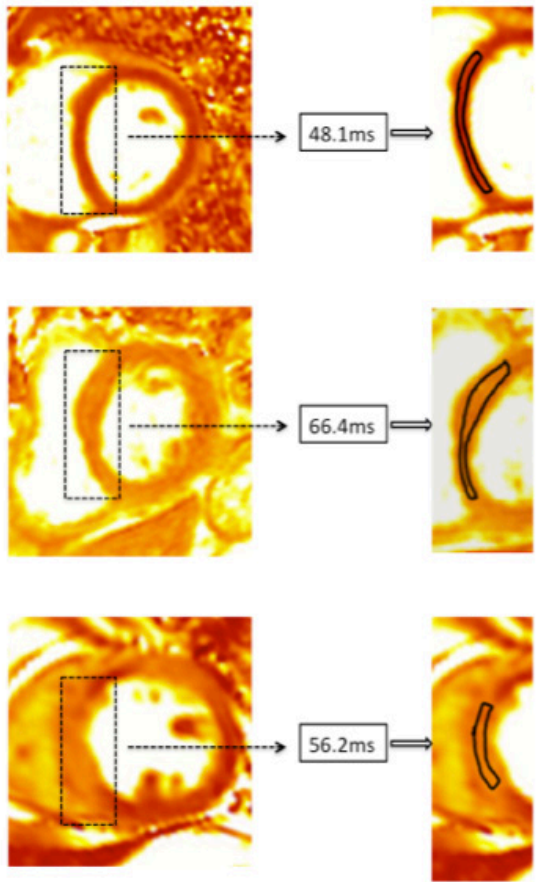

Figure 1 Post-USPIO R2* and T2 maps for a healthy volunteer (A), patient with cardiac transplant $(B)$ and myocardial infarction (C). Myocardial oedema is displayed as brighter signal in the T2 colour map corresponding to higher T2 value. Cardiac transplant myocardium (B) displays no USPIO accumulation but high T2, in contrast to USPIO accumulation and high T2 in myocardial infarction (C). USPIO, ultra-small superparamagnetic particles of iron oxide.

following the baseline magnetic resonance scan over $15 \mathrm{~min}$ using a concentration of $2-8 \mathrm{mg} / \mathrm{mL}$, diluted in $0.9 \%$ saline or $5 \%$ dextrose. Haemodynamic monitoring was conducted throughout, and participants were observed for a further $30 \mathrm{~min}$ to ensure no hypersensitivity reactions.

\section{Image analysis}

All T2*-weighted multigradient-echo images for each patient were analysed using Circle CVI software (Circle CVI42, Canada). An experimentally determined threshold used in previous work $^{6}$ for the coefficient of determination $\left(r^{2}>0.85\right)$ was used to exclude data that did not have an acceptable exponential decay when signal intensity (SI) was plotted against echo time. Individual images affected by artefact were excluded. The inverse of the mean $\mathrm{T}^{*}(\mathrm{R} 2 *)$ for each region of interest (ROI) was then calculated to assess the uptake of USPIO, where the higher the value, the greater the USPIO accumulation.

T2 maps, ventricular volume and functional analyses were also performed using Circle CVI software (Circle CVI42, Canada). T2 and T2* data were collected immediately prior to USPIO administration. USPIO-enhanced T2* data were collected 24-25 hours following ferumoxytol administration. ROIs were drawn on T2 and T2* images in the septum at midcavity level between anterior and inferior RV insertion points (see figure 1). Septal myocardial regions only were selected to reduce influence of artefact caused by nearby stomach or lung. ${ }^{21}$

\section{Histology}

Myocardial tissue samples were obtained from one patient with cardiac transplantation undergoing surveillance biopsies without suspicion of transplant rejection. USPIO was administered 24 hours prior to the biopsy, and a trucut myocardial biopsy sample was taken from the myocardium. The biopsy sample was fixed in formalin, embedded in paraffin, sectioned and stained to look at architecture (H\&E), accumulation and distribution of USPIO (Prussian Blue) and macrophages (CD68).

\section{Sample size and statistical analysis}

Previous work has shown the change in R2* due to USPIO in 'healthy' myocardium (remote from the site of myocardial infarction) to be $40 \pm 11 \mathrm{~s}^{-1}$ at 24 hours following USPIO. ${ }^{6}$ Assuming the change due to USPIO is similar in truly healthy myocardium, a sample size of 10 in each group is needed to detect an effect size of $20 \mathrm{~s}^{-1}$ due to USPIO (ie, an increase of $50 \%$ ) with $80 \%$ power and significance level of 0.05 .

All statistical analysis was performed with GraphPad Prism, version 6 (GraphPad Software, San Diego, California, USA). Shapiro-Wilk or D'Agostino and Pearson tests were used to test normality of distribution. To compare participant characteristics, USPIO uptake and myocardial oedema in patients and volunteers, R2* and $\mathrm{T} 2$ values were compared using $\chi^{2}$, unpaired t-tests and Mann-Whitney tests as appropriate depending on normality distribution. To compare results at 3 months with baseline, paired t-test and Wilcoxon test was used. Statistical significance was defined as two sided $\mathrm{p}<0.05$.

\section{RESULTS}

Ten volunteers and 11 patients with cardiac transplantation were recruited. One volunteer was excluded from analysis due to the presence of LGE as described previously. ${ }^{21}$ At the time of recruitment, all transplant patients were assessed to be clinically well with no firm evidence of allograft rejection based on clinical history, examination and routine blood samples. One patient with prior cardiac transplant was excluded due the finding of LGE consistent with myocardial infarction that may influence the USPIO enhancement data. ${ }^{6}$ No other patient with cardiac transplant displayed LGE. Five of the 10 cardiac transplant patients returned at 3 months for repeat imaging. Administration of ferumoxytol was well tolerated with no adverse reactions reported during or immediately after administration in any of the participants.

Volunteers were predominantly female and patients with cardiac transplant were predominantly male (table 1). Transplant patients had smaller indexed end-diastolic ventricular volumes $(\mathrm{p}<0.01)$ and higher 


\begin{tabular}{|c|c|c|}
\hline & $\begin{array}{l}\text { Healthy } \\
\text { volunteers }\end{array}$ & $\begin{array}{l}\text { Patients with } \\
\text { transplant }\end{array}$ \\
\hline Number & 9 & 10 \\
\hline Female & 6 & $1^{*}$ \\
\hline Age (years) & $52(45.5-61.5)$ & $60(52.75-64.5)$ \\
\hline $\begin{array}{l}\text { Time since transplantation } \\
\text { (months) }\end{array}$ & & $59(19-159)$ \\
\hline Body mass Index $\left(\mathrm{kg} / \mathrm{m}^{2}\right)$ & $22.9(20.1-26.9)$ & $25.9(24.0-27.9)$ \\
\hline $\begin{array}{l}\text { Left ventricular end-diastolic } \\
\text { volume }\left(\mathrm{mL} / \mathrm{m}^{2}\right)\end{array}$ & $80.9 \pm 10.4$ & $62.9 \pm 15.5^{\star *}$ \\
\hline $\begin{array}{l}\text { Left ventricular ejection } \\
\text { fraction }(\%)\end{array}$ & $63.6 \pm 4.9$ & $65.0 \pm 7.1$ \\
\hline \multicolumn{3}{|l|}{ Blood tests } \\
\hline White cell count $\left(\times 10^{9} / \mathrm{L}\right)$ & $6.5 \pm 1.5$ & $6.1 \pm 2.5$ \\
\hline C reactive protein (mg/L) & $1.9 \pm 2.0$ & $2.1 \pm 2.1$ \\
\hline Plasma troponin (ng/L) & $3.1 \pm 2.8$ & $14.1 \pm 20.9^{*}$ \\
\hline
\end{tabular}

Mean \pm SD or median (IQR).

${ }^{*} \mathrm{p}<0.05,{ }^{* *} \mathrm{p}<0.01$ (compared with volunteers).

baseline plasma high-sensitivity troponin concentrations $(p<0.05)$. There were more males in the transplant group $(p<0.05)$, but there were no other differences between groups at baseline.

\section{T2 and R2* mapping}

Patients with cardiac transplantation had a higher T2 value than volunteers $(53.8 \pm 5.2$ vs $48.6 \pm 1.9 \mathrm{~ms}$, respectively, $\mathrm{p}=0.003$; figures 1 and 2 and table 2 ). There were

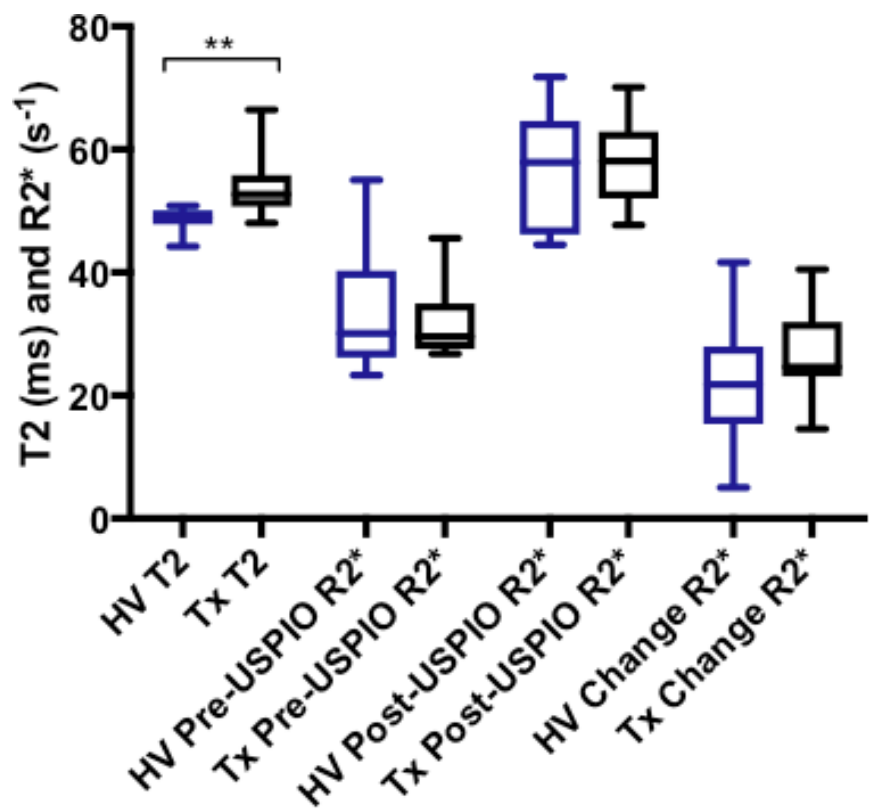

Figure 2 Comparison of T2 (ms) and $\mathrm{R} 22^{*}\left(\mathrm{~s}^{-1}\right)$ measurements at baseline in healthy volunteers (HV) and cardiac transplant patients (Tx). Transplant patients have higher T2 than healthy volunteers, but no other significant differences exist between groups $\left({ }^{* *} \mathrm{p}<0.01\right)$. USPIO, ultra-small superparamagnetic particles of iron oxide.
Table 2 Septal myocardial R2* and T2 values in healthy volunteers and patients with cardiac transplantation

\begin{tabular}{lll}
\hline & $\begin{array}{l}\text { Patients with } \\
\text { transplant }\end{array}$ & $\begin{array}{l}\text { Healthy } \\
\text { volunteers }\end{array}$ \\
\hline Pan-myocardial pre-USPI0 R2* $\left(\mathrm{s}^{-1}\right)$ & $31.6 \pm 5.9$ & $34.0 \pm 10.1$ \\
Pan-myocardial post-USPI0 R2* $\left(\mathrm{s}^{-1}\right)$ & $58.2 \pm 7.5$ & $56.0 \pm 10.2$ \\
Pan-myocardial $\triangle \mathrm{R}^{*}\left(\mathrm{~s}^{-1}\right)$ & $26.6 \pm 7.3$ & $22.0 \pm 10.4$ \\
T2 (ms) & $53.8 \pm 5.2^{*}$ & $48.6 \pm 1.9$ \\
\hline
\end{tabular}

Mean \pm SD.

${ }^{*} \mathrm{p}<0.01$ (compared with volunteers).

USPIO, ultra-small superparamagnetic particles of iron oxide.

no differences in $\mathrm{R}^{*} *$ between patients with cardiac transplantation and volunteers at baseline $(31.6 \pm 5.9$ vs $\left.34.0 \pm 10.1 \mathrm{~s}^{-1}, \mathrm{p}=0.84\right)$, at 24 hours after USPIO administration $\left(58.2 \pm 7.5\right.$ vs $\left.56.0 \pm 10.2 \mathrm{~s}^{-1}, \mathrm{p}=0.60\right)$ or in the magnitude of change in R2* due to USPIO (26.6 \pm 7.3 vs $22.0 \pm 10.4 \mathrm{~s}^{-1}, \mathrm{p}=0.28$; figures 1 and 2 and table 2$)$.

There were no differences in either T2 $(52.7 \pm 2.8$ vs $52.12 \pm 3.4 \mathrm{~ms}, \mathrm{p}=0.80$ ) or the change in $\mathrm{R} 2 *$ due to USPIO (29.42 \pm 8.14 vs $25.8 \pm 7.8 \mathrm{~s}^{-1}, \mathrm{p}=0.43$ ) between baseline and 3 months in patients with cardiac transplant (figure 3). The myocardial biopsy 24 hours after USPIO administration revealed normal myocyte architecture with no evidence of increased numbers of inflammatory macrophages or the presence of USPIO (figure 4).

\section{Cardiac transplant patient with recently suspected allograft rejection}

One patient was recruited to the study 3 days following admission with non-specific malaise. This was the only patient in our cohort that had a previous history of previous acute cellular rejection (2 years before). On this occasion, the myocardial biopsy showed grade 1a rejection (ie, no evidence of recurrence of rejection or cellular infiltration), and the patient was reassured but subsequently recruited to the study. This patient had the highest plasma high-sensitivity troponin I in the cohort at $71 \mathrm{ng} / \mathrm{L}$. Other blood tests were normal including white cell count $\left(4.3 \times 10^{9} / \mathrm{L}\right)$ and $\mathrm{C}$ reactive protein $(<1 \mathrm{mg} / \mathrm{L})$. The T2 value for this subject was the highest in our cohort at $66.4 \mathrm{~ms}$ (16-segment average of 61.8 $\mathrm{ms}) . \mathrm{R} 2 *$ was $26.7 \mathrm{~s}^{-1}$ at baseline and $50.6 \mathrm{~s}^{-1} 24$ hours after USPIO: change in $\mathrm{R}^{*}$ was $23.8 \mathrm{~s}^{-1}$. A reanalysis of the data
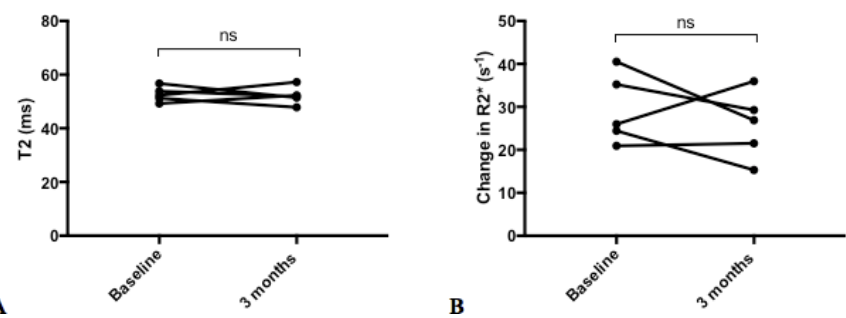

Figure 3 Repeated imaging in patients with cardiac transplant. There were no differences in myocardial T2 (ms) (A) or the change in $\mathrm{R}^{*}\left(\mathrm{~s}^{-1}\right)(\mathrm{B})$ due to USPIO between time points $(n=5)$. 


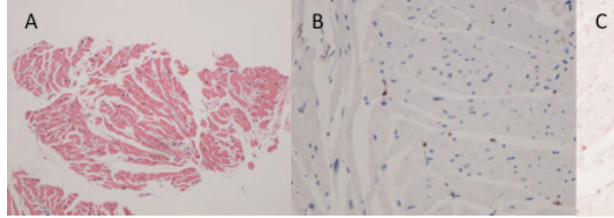

Figure 4 Biopsy from patient with cardiac transplant. (A) H\&E $(\times 100)$ staining showing normal myocyte architecture with no evidence of significant inflammatory cell infiltration. (B) CD68 staining $(\times 200)$ showing only very few scattered macrophages (brown). (C) Prussian blue staining showing no evidence of iron.

censoring this patient from analysis continued to show a higher T2 value in patients with cardiac transplantation than volunteers ( $52.4 \pm 2.8$ vs $48.6 \pm 1.9 \mathrm{~ms}, \mathrm{p}=0.0056)$. This patient did not undergo gadolinium-enhanced imaging at the request of the attending clinician due to mild renal impairment.

\section{DISCUSSION}

For the first time, we report the combined assessment of T2 mapping and USPIO-enhanced T2* MRI in patients with prior cardiac transplantation and healthy volunteers. This technique is feasible, safe and well tolerated in this group of patients. We demonstrate that while myocardial T2 is increased, there is no evidence of ongoing or chronic cellular inflammatory uptake as detected by USPIO enhancement. Further assessments of these measures are now needed in patients with acute cardiac transplant rejection.

USPIO-enhanced T2* MRI has previously been used in man to assess cardiovascular inflammation in a range of different conditions, ${ }^{5-8} 11$ and preclinical models suggest this technique may be useful in assessing human patients with transplant rejection. ${ }^{413-17}$ In our cohort of stable patients with cardiac transplantation, we did not detect greater USPIO enhancement within the myocardium, but this contrasted with the increased measures of myocardial $\mathrm{T} 2$ found in transplant patients compared with volunteers.

Why did we detect higher T2 values in our cohort of transplant patients compared with controls in the absence of USPIO accumulation? There are several plausible explanations. First, chronic residual low-grade inflammation or oedema without tissue-resident macrophage infiltration is possible, perhaps from lymphocytes that do not take up USPIO. In the absence of histological data, we cannot confirm the presence of lymphocytes, but in a stable group of patients who are clinically well, this explanation appears unlikely. Second, higher T2 values in the myocardium of cardiac allografts may simply be due to increased water content, possibly related to expansion of the myocardial vasculature and blood content. The finding of greater measures of R2* (both at baseline and after USPIO), which measures iron within the blood pool, would support this mechanism. This explanation may suggest intriguing differences in the behaviour of resting myocardial vascular integrity in patients with stable cardiac allografts. Third, the relatively low field strength of the MRI scanner (1.5T) may be contributory since it has poorer sensitivity in detecting sparse myocardial USPIO compared with higher field strength 3T scanners. However, in our single myocardial biopsy, substantial numbers of macrophages were not evident. Finally, a possible explanation for greater measures of T2 in cardiac allografts may in fact reflect a change in myocyte architecture caused by myocardial fibrosis or scarring. High T2 value is usually solely attributed to myocardial oedema but has previously been found to inversely correlate with $\mathrm{LV}$ function in patients with dilated cardiomyopathy that did not have any evidence of inflammation or other reasons for myocardial oedema. ${ }^{22}$ This may suggest that in this cohort higher T2 values reflect myocardial fibrosis, similar to results using T1 mapping techniques. Recently published data in a similar cohort of patients found elevation of T2 and T1 values in all transplanted patients, even without history of cardiac rejection, and the authors suggest this is due to greater myocardial oedema and interstitial fibrosis at baseline. ${ }^{23}$ However, we contend that elevated T2 in the myocardium of cardiac allografts in this cohort may reflect intrinsic changes to the cellular architecture, such as the presence of myocardial fibrosis and not myocardial oedema. As such, it may prove to be a further imaging biomarker of myocardial fibrosis. Crucially, we lacked adequate access to sufficient histology or T1 mapping in order to prove whether subtle myocardial fibrosis, not detected by LGE imaging, is a causative factor in the high T2 value found. Most patients had undergone transplantation many years ago (see table 1) so using historical postoperative surveillance biopsies would not provide an accurate assessment of current myocyte architecture.

Irrespective of the aetiology, greater T2 in stable transplant patients is an interesting finding reported recently that we have now confirmed and warrants further investigation. Previous small studies have found that T2-weighted and T2 mapping MRI assists in diagnosis and prognosis estimation in patients with cardiac transplant rejection. ${ }^{24-26}$ Following on from these studies, the DRAGET (Detection of Acute Graft Rejection in Heart Transplant Patients by Estimation of T2) study, ${ }^{27}$ a large multicentre multinational study, is now prospectively recruiting patients in the first year after transplantation to assess the performance of T2 mapping in diagnosing transplant rejection. The findings of this study are awaited with interest, but clearly if the $\mathrm{T} 2$ value in myocardium of stable cardiac allografts is raised, this increases the likelihood of false-positive diagnoses of allograft rejection and ultimately reduces the specificity and precision of the test.

One patient had very high myocardial T2 and deserves special mention. This was the only patient with a previous history of biopsy proven acute cellular rejection 2 years previously. At the time of recruitment, the patient was recovering from a non-specific illness, and recurrent episode of rejection had been discounted on the basis 
of negative endomyocardial biopsies 3 days previously. Based on the studies mentioned previously, myocardial T2 value of this magnitude suggests severe rejection. Serial imaging with T2 mapping, both prior and subsequent to this episode, would have been useful in determining the time course and fluctuation in $\mathrm{T} 2$ to assess whether it is chronically elevated, which may suggest stable myocardial fibrosis or not. This patient in fact had T1 maps acquired as part of imaging biobank retention (the only one in our study), and septal values were elevated at $1018 \mathrm{~ms}$ (normal values $950 \pm 21 \mathrm{~ms}$ ) ${ }^{28}$ Clearly, we cannot make generalisations from one patient, but this provides further support to the argument that high T2 may reflect myocardial fibrosis.

Whether this patient had allograft rejection (with a negative biopsy) remains uncertain, but a clinical improvement was made with no new changes to medical therapy, making an episode of rejection is less likely. There was certainly no evidence for USPIO uptake or significant macrophage infiltration. If myocardial fibrosis and active rejection or inflammation is not the cause of elevated T2, then why was the T2 value so high in this patient? A high T2 value of this magnitude in the absence of rejection would cast doubt on the precision of $\mathrm{T} 2$ mapping to diagnose and exclude cardiac allograft rejection and diminish its potential utility in clinical practice. The DRAGET study will be well placed to answer this. ${ }^{27}$

There are some limitations that deserve mention. Our numbers were small but sufficient for a pilot study testing the feasibility of USPIO-enhanced MRI in this population. From our recent work in patients with myocardial infarction, we know that this technique works and is capable of identifying and tracking myocardial macrophage accumulation macrophage. ${ }^{5}$ In this study, myocardial macrophage infiltration is either not a chronic feature of stable transplants or at levels too low to be detected.

We intended to recruit a range of patients with cardiac transplantation, from chronic stable patients, to patients with suspected allograft rejection. Unfortunately, we were only able to recruit one patient with recently suspected transplant rejection, and this was excluded clinically, with the remaining patients being stable and well. Small study numbers and absence of patients with florid transplant rejection clearly limit our findings, and this technique now needs to be tested in patients with proven acute transplant rejection.

In conclusion, we have shown that stable patients with cardiac transplantation have greater measures of myocardial T2, which may suggest myocardial oedema or fibrosis, compared with control volunteers. Ferumoxytol-enhanced MRI does not add clinically relevant information in this group of stable patients, and it remains to be determined whether this technique may be able to identify macrophages in the setting of proven acute cellular transplant rejection.

Acknowledgements This work was supported by the Chief Scientist Office (ETM/266). SA and DEN are supported by the British Heart Foundation (FS/12/83;
$\mathrm{CH} / 09 / 002)$. DEN is the recipient of a Wellcome Trust Senior Investigator Award (WT103782AIA). MRD is supported by the British Heart Foundation ( $\mathrm{FS} / 14 / 78 / 31020$ ) and is the recipient of the Sir Jules Thorn Award for Biomedical Research 2015. Wellcome Trust Clinical Research Facility and the Clinical Research Imaging Centre are supported by NHS Research Scotland (NRS) through NHS Lothian.

Contributors CGS, SA, SS and DEN designed the study, collected and analysed, data and drafted the manuscript. TJM, CG, MRD, VJ and WW analysed and interpreted data and drafted the manuscript. MRD, JRP, SKP, RSG, SM and PH designed the study and drafted the manuscript. All authors read and approved the manuscript.

Funding This work was supported by the Chief Scientist Office (ETM/266). SA, MRD and DEN are supported by the British Heart Foundation (FS/12/83; FS/14/78/31020; $\mathrm{CH} / 09 / 002)$. DEN is the recipient of a Wellcome Trust Senior Investigator Award (WT103782AIA).

Competing interests None declared.

Patient consent for publication Not required.

Provenance and peer review Not commissioned; internally peer reviewed.

Data availability statement All data relevant to the study are included in the article or uploaded as supplementary information.

Open access This is an open access article distributed in accordance with the Creative Commons Attribution 4.0 Unported (CC BY 4.0) license, which permits others to copy, redistribute, remix, transform and build upon this work for any purpose, provided the original work is properly cited, a link to the licence is given, and indication of whether changes were made. See: https://creativecommons.org/ licenses/by/4.0/.

ORCID iD

Colin G Stirrat http://orcid.org/0000-0001-9688-5517

\section{REFERENCES}

1. Lund LH, Khush KK, Cherikh WS, et al. The registry of the International Society for heart and lung transplantation: Thirty-fourth adult heart transplantation Report-2017; focus theme: allograft ischemic time. J Heart Lung Transplant 2017;36:1037-46.

2. Mannon RB. Macrophages: contributors to allograft dysfunction, repair, or innocent bystanders? Curr Opin Organ Transplant 2012;17:20-5.

3. Stewart S, Winters GL, Fishbein MC, et al. Revision of the 1990 working formulation for the standardization of Nomenclature in the diagnosis of heart rejection. J Heart Lung Transplant 2005;24:1710-20.

4. Wu YL, Ye Q, Eytan DF, et al. Magnetic resonance imaging investigation of macrophages in acute cardiac allograft rejection after heart transplantation. Circ Cardiovasc Imaging 2013;6:965-73.

5. Stirrat CG, Alam SR, MacGillivray TJ, et al. Ferumoxytol-enhanced magnetic resonance imaging assessing inflammation after myocardial infarction. Heart 2017;103:1528-35.

6. Alam SR, Shah ASV, Richards J, et al. Ultrasmall superparamagnetic particles of iron oxide in patients with acute myocardial infarction: early clinical experience. Circ Cardiovasc Imaging 2012;5:559-65.

7. Yilmaz A, Dengler MA, van der Kuip $\mathrm{H}$, et al. Imaging of myocardial infarction using ultrasmall superparamagnetic iron oxide nanoparticles: a human study using a multi-parametric cardiovascular magnetic resonance imaging approach. Eur Heart $J$ 2013;34:462-75.

8. Richards JMJ, Semple SI, MacGillivray TJ, et al. Abdominal aortic aneurysm growth predicted by uptake of ultrasmall superparamagnetic particles of iron oxide: a pilot study. Circ Cardiovasc Imaging 2011;4:274-81.

9. McBride OMB, Berry C, Burns P, et al. MRI using ultrasmall superparamagnetic particles of iron oxide in patients under surveillance for abdominal aortic aneurysms to predict rupture or surgical repair: MRI for abdominal aortic aneurysms to predict rupture or surgery-the MA $\left.{ }^{(3)}\right)$ RS study. Open Heart 2015;2:e000190.

10. Trivedi RA, Mallawarachi $\mathrm{C}, \mathrm{U}-\mathrm{King}-\mathrm{Im} \mathrm{J}-\mathrm{M}$, et al. Identifying inflamed carotid plaques using in vivo USPIO-Enhanced MR imaging to label plaque macrophages. Arterioscler Thromb Vasc Biol 2006;26:1601-6.

11. Tang TY, Howarth SPS, Miller SR, et al. The atheroma (atorvastatin therapy: effects on reduction of macrophage activity) StudyEvaluation using ultrasmall superparamagnetic iron oxide- 
enhanced magnetic resonance imaging in carotid disease. JAC 2009;53:2039-50.

12. Ruehm SG, Corot C, Vogt $P$, et al. Magnetic resonance imaging of atherosclerotic plaque with ultrasmall superparamagnetic particles of iron oxide in hyperlipidemic rabbits. Circulation 2001;103:415-22.

13. Johansson L, Johnsson C, Penno E, et al. Acute cardiac transplant rejection: detection and grading with MR imaging with a blood pool contrast Agent-Experimental study in the rat. Radiology 2002;225:97-103.

14. Penno E, Johnsson $\mathrm{C}$, Johansson L, et al. Comparison of ultrasmall superparamagnetic iron oxide particles and low molecular weight contrast agents to detect rejecting transplanted hearts with magnetic resonance imaging. Invest Radiol 2005;40:648-54

15. $\mathrm{YL} \mathrm{W}, \mathrm{Ye} Q$, Sato $\mathrm{K}$, et al. Noninvasive evaluation of cardiac allograft rejection by cellular and functional cardiac magnetic resonance. JCMG 2009;2:731-41.

16. Kanno S, Wu Y-JL, Lee PC, et al. Macrophage accumulation associated with rat cardiac allograft rejection detected by magnetic resonance imaging with ultrasmall superparamagnetic iron oxide particles. Circulation 2001;104:934-8.

17. Alam SR, Tse GH, Stirrat C, et al. Nanoparticle enhanced MRI scanning to detect cellular inflammation in experimental chronic renal allograft rejection. Int J Mol Imaging 2015;2015:1-8.

18. Guo Y, Chen W, Wang W, et al. Simultaneous diagnosis and gene therapy of immuno-rejection in rat allogeneic heart transplantation model using a T-cell-targeted theranostic nanosystem. ACS Nano 2012;6:10646-57.

19. Thavendiranathan P, Walls M, Giri S, et al. Improved detection of myocardial involvement in acute inflammatory cardiomyopathies using T2 mapping. Circulation: Cardiovascular Imaging 2012;5:102-10.
20. Giri S, Chung Y-C, Merchant A, et al. T2 quantification for improved detection of myocardial edema. J Cardiovasc Magn Reson 2009;11.

21. Stirrat CG, Alam SR, MacGillivray TJ, et al. Ferumoxytol-enhanced magnetic resonance imaging methodology and normal values at 1.5 and 3T. J Cardiovasc Magn Reson 2016;18.

22. Nishii T, Kono AK, Shigeru M, et al. Cardiovascular magnetic resonance T2 mapping can detect myocardial edema in idiopathic dilated cardiomyopathy. Int J Cardiovasc Imaging 2014;30:65-72.

23. Dolan RS, Rahsepar AA, Blaisdell J, et al. Multiparametric Cardiac Magnetic Resonance Imaging Can Detect Acute Cardiac Allograft Rejection After Heart Transplantation. JACC: Cardiovascular Imaging 2019;12:1632-41.

24. Usman AA, Taimen $\mathrm{K}$, Wasielewski M, et al. Cardiac magnetic resonance T2 mapping in the monitoring and follow-up of acute cardiac transplant rejection: a pilot study. Circ Cardiovasc Imaging 2012;5:782-90.

25. Marie PY, Carteaux JP, Escanye JM, et al. Detection and prediction of acute heart transplant rejection with the myocardial T2 determination provided by a black-blood magnetic resonance imaging sequence. J Heart Lung Transplant 2001;20:193-4.

26. Bonnemains L, Villemin T, Escanye J-M, et al. Diagnostic and prognostic value of MRI T2 quantification in heart transplant patients. Transp/ Int 2014;27:69-76.

27. Bonnemains L, Cherifi A, Girerd N, et al. Design of the DRAGET Study: a multicentre controlled diagnostic study to assess the detection of acute rejection in patients with heart transplant by means of $\mathrm{T}_{2}$ quantification with $\mathrm{MRI}$ in comparison to myocardial biopsies. BMJ Open 2015;5:e008963.

28. Dabir D, Child N, Kalra A, et al. Reference values for healthy human myocardium using a T1 mapping methodology: results from the International T1 multicenter cardiovascular magnetic resonance study. J Cardiovasc Magn Reson 2014;16. 